\title{
ON SCRAMBLED SETS FOR CHAOTIC FUNCTIONS
}

\author{
A. M. BRUCKNER AND THAKYIN HU
}

\begin{abstract}
Some recent research has raised questions concerning the possible sizes of scrambled sets for chaotic functions. We answer these questions by showing that a scrambled set can have full measure, but cannot be residual although a scrambled set can be second category in every interval. We also indicate relationships that exist between chaotic functions and transitive functions.
\end{abstract}

1. Introduction. A number of problems involving the way a process evolves with time give rise to rather simple looking difference equations. This may happen, for example, when one wishes to estimate the size of some future generation of a population purely on the basis of its present size. The model $x_{n+1}=f\left(x_{n}\right)$ indicating the size $x_{n+1}$ of the $(n+1)$ st generation in terms of the $n$th can be studied in terms of a function $f$ mapping the interval $[0,1]$ onto itself and analyzing the behavior of the iterates $x, f(x), f(f(x)), \ldots$ Often $f$ vanishes at the end-points of the interval and achieves a single maximum at some interior point of $[0,1]$. The interested reader may consult [LY and VSK] for discussions of such problems and for extensive reference lists.

An investigator dealing with such problems may hope for essential stability of the iterates; that is, that the sequence $x, f(x), f(f(x)), \ldots$ converges to some fixed point of $f$, indicating that the population will stabilize at some size independent of $x(x \neq 0,1)$. If that fails, the investigator would at least hope for predictability: if the initial population is $x_{0}$, which the investigator estimates reasonably accurately as $y_{0}$, he would hope that the sequence of iterates remain close together. In that case, a slight error in the estimate for the initial population will result in no more than a slight error in the estimates of future generations. Unfortunately, it often happens that neither of these wishes is satisfied. The investigator usually observes various sorts of chaotic behavior, all of which are predictable from the model.

In recent years, a number of authors have studied various sorts of chaotic behavior of functions of the type we mentioned. They have also asked whether certain even more chaotic behavior is possible. In the present paper we answer some of their questions, and discuss other related types of behavior. In $\S 2$, we provide the necessary definitions, review some of the recent work that motivated this paper and provide some results that we shall need in the sequel. In $\S 3$, we obtain affirmative answers to questions posed in [S2]. These questions relate to the size (in terms of Lebesgue measure) possible of so-called scrambled sets. Then in $\S 4$, we study the

Received by the editors August 20, 1985 and, in revised form, January 6, 1986.

1980 Mathematics Subject Classification (1985 Revision). Primary 26A18, 54H20.

Key words and phrases. Chaotic functions, scrambled sets, transitive (or nomadic) functions.

The first author was supported in part by a grant from the NSF and the second author was supported in part by a grant from the NSC of the Republic of China. 
analogues of these questions when the size of a set is measured by (Baire) category rather than measure. We also indicate the relationships that exist between two types of behavior, "chaotic" and "nomadic" (or "transitive").

2. Preliminaries. Let $f$ be a function mapping the interval $I_{0}=[0,1]$ onto itself. Let $x \in I_{0}$. By the orbit of $x$ (under $f$ ), we mean the sequence $\left\{f^{n}(x)\right\}_{n=0}^{\infty}$, where $f^{0}(x)=x, f^{1}(x)=f(x)$, and in general $f^{n+1}(x)=f\left(f^{n}(x)\right), n=0,1, \ldots$ We shall use the notation $O(x)$ for the set that is the range of the sequence $\left\{f^{n}(x)\right\}$. If $I \subseteq I_{0}$ is an interval, we will denote by $O(I)$ the orbit of the interval $I$ (under $f$ ); i.e., $O(I)=\bigcup_{n=0}^{\infty} f^{n}(I)$. Similarly, the inverse orbits of $x$ and of $I$ (under $f$ ) are defined by $O^{-1}(x)=\left\{y: f^{n}(y)=x\right.$ for some $\left.n=0,1,2, \ldots\right\}$ and $O^{-1}(I)=$ $\left\{O^{-1}(x): x \in I\right\}$.

We shall be concerned primarily with so-called chaotic functions and their scrambled sets. While these notions have been studied by a number of authors, no entirely consistent use of the terms has evolved. The definitions we give below represent a strong form of chaotic behavior suitable for our purposes.

Let $f$ be a continuous function mapping $I_{0}$ onto $I_{0}$.

DEFINITION 2.1. A set $S \subseteq I_{0}$ is called scrambled for $f$ provided for every $x, y \in S$ with $x \neq y$,

$$
\limsup _{n \rightarrow \infty}\left|f^{n}(x)-f^{n}(y)\right|=1
$$

and

$$
\liminf _{n \rightarrow \infty}\left|f^{n}(x)-f^{n}(y)\right|=0 .
$$

DEFINITION 2.2. The function $f$ is called chaotic if there is an uncountable scrambled set for $f$.

REMARK. Other authors have replaced (1) with the weaker requirement that $\limsup _{n \rightarrow \infty}\left|f^{n}(x)-f^{n}(y)\right|>0$, but at the same time have required something about the behavior of points in their scrambled sets vis-à-vis periodic points. Kan $[\mathbf{K}]$ uses the term "extremally scrambled" when (1) is satisfied. Because of requirement (1), we restrict ourselves in $\S \S 3$ and 4 to functions mapping $I_{0}$ onto $I_{0}$. The recent articles $[\mathbf{K}, \mathbf{S 1}, \mathbf{S 2}]$ give examples of chaotic functions whose scrambled sets have positive measure or full outer measure. The question arises naturally [S2] whether a scrambled set can have full measure. We shall answer this question affirmatively by an example in $\S 3$.

We shall also make use of functions exhibiting a type of related behavior.

DEFINITION 2.3. A function $f: I_{0} \rightarrow I_{0}$ is called nomadic if there exists $x \in I_{0}$ such that $O(x)$ is dense.

Other authors have used other terms (e.g. "transitive") to express this phenomenon. Since we shall make use of results in [BCR], where the term "nomadic" was used, we retain that term.

PROPOSITION 2.4. The function $f: I_{0} \rightarrow I_{0}$ is nomadic if and only if $O(I)$ is dense for every interval $I \subseteq I_{0}$.

PROOF. If $f$ is nomadic, then it is clear that $O(I)$ is dense for each interval $I \subseteq I_{0}$.

To prove the converse, observe first that our hypothesis implies that if $J$ is an arbitrary interval in $I_{0}$, then $O^{-1}(J)$ is dense. Now let $\left\{J_{k}\right\}_{k=1}^{\infty}$ be a countable 
base for the topology of $[0,1]$. Let $A=\bigcap_{k=1}^{\infty} O^{-1}\left(J_{k}\right)$. Since $O^{-1}\left(J_{k}\right)$ is dense and open for every $k, A$ is a dense set of type $G_{\delta}$ in $[0,1]$. If $x \in A$, then $O(x)$ is dense. Thus $f$ is nomadic.

3. Scrambled sets of full measure. We turn now to a construction of a function $g$ possessing a scrambled set of full measure. The function $g$ is strictly increasing on $\left[0, \frac{1}{2}\right]$ and strictly decreasing on $\left[\frac{1}{2}, 1\right]$. It provides affirmative answers to two questions of Smital [S2].

We shall make use of a function which has been studied by several authors. We begin with a brief review of this function, indicating the properties of this function which we shall need. Define $f: I_{0} \rightarrow I_{0}$ by

$$
f(x)= \begin{cases}2 x & \text { if } 0 \leq x \leq \frac{1}{2} \\ 2(1-x) & \text { if } \frac{1}{2} \leq x \leq 1\end{cases}
$$

It is convenient to consider the binary expansion of points in $[0,1]$. One can then verify easily (see $[\mathbf{B C R}]$ ) that:

(1) If $x=.0 x_{2} x_{3} x_{4} \cdots$ (binary expansion), then $f(x)=x_{2} x_{3} x_{4} \cdots$

(b) If $x=.1 x_{2} x_{3} x_{4} \cdots$ (binary expansion), then $f(x)=._{2} y_{3} y_{4} \cdots$, where $y_{i}=0$ if $x_{i}=1$ and $y_{i}=1$ if $x_{i}=0$.

(c) If every finite sequence of 0 's and 1's appears in the binary expansion of $x$, then $x$ has a dense orbit. Thus, almost every $x \in[0,1]$ has a dense orbit.

Smital $[\mathbf{S 1}]$ has shown that $f$ has a scrambled set of full outer measure, but that any measurable scrambled set for $f$ must have zero measure.

Our construction of a function $g$ possessing a scrambled set of full measure will involve a number of steps. We first show that the function $f$ has a scrambled set $S$ of Borel type $G_{\delta \sigma}$ that has cardinality of the continuum in every interval. A suitable transformation then results in the function $g$. The main problem in obtaining $S$ arises from the following considerations. If $S$ is a scrambled set for $f$ and $x \neq y$ are in $S$, then the binary expansions for $x$ and $y$ must contain arbitrarily long strings of 0 's and 1's (see (a) and (b) above). These strings must be positioned properly so that the definition of scrambled set is satisfied for $x$ and $y$. These requirements must be achieved by every pair $(x, y)$ in $S$. We shall achieve this by representing certain points in $I_{0}$ by "wedges."

THEOREM 3.1. There exist a continuous function $g: I_{0} \rightarrow I_{0}$ such that $g$ is increasing on $\left[0, \frac{1}{2}\right]$ and $g$ is decreasing on $\left[\frac{1}{2}, 1\right]$, and a corresponding scrambled set $T$ with $T \in F_{\sigma}$ and $\lambda T=1$.

PROOF. We shall prove the theorem by the following five major steps:

(1) Let $L$ be the lattice of pairs of positive integers and $0<\xi<1$ be a fixed irrational number. Let $\Lambda$ be the family of positive, finite slopes of all rays emanating from $(0, \xi)$, lying in the first quadrant, and containing no points of $L$. If $r, s \in \Lambda$ with $r<s$, let $W_{r s}=\{(x, y) \in L: r x+\xi<y<s x+\xi\}$. Let $L$ be enumerated diagonally, $l_{1}, l_{2}, \ldots$, as follows:

$$
(1,1),(1,2),(2,1),(1,3),(2,2),(3,1),(1,4),(2,3),(3,2), \ldots
$$

Let $\mathcal{W}$ be the system of a finite union of wedges. Thus an element of $\mathcal{W}$ consists of the union of a finite number of "wedges,"

$$
W_{r_{1} s_{1}}, W_{r_{2} s_{2}}, \ldots, W_{r_{n} s_{n}}, \quad r_{1}<s_{1}<r_{2}<s_{2}<\cdots<r_{n}<s_{n} .
$$


Now to each $W \in \mathcal{W}$, we associate that number $x_{W} \in[0,1]$ whose binary expansion has a 1 in the $i$ th position if and only if the $i$ th lattice point $l_{i}$ is in $W$. Let $S=\left\{x_{W}: W \in \mathcal{W}\right\}$. Then

(i) $\operatorname{Card} S=c$.

(ii) For any finite set of integers $i_{1}<i_{2}<\cdots<i_{n}$, there exists some $W \in \mathcal{W}$ containing $l_{i_{1}}, l_{i_{2}}, \ldots, l_{i_{n}}$ corresponding to $i_{1}, i_{2}, \ldots, i_{n}$ but no other lattice point $l_{j}$ with $j \leq i_{n}$. It follows that $S$ is dense in $[0,1]$. Since a slight variant of $W$ in (ii) leads to a different $W^{*} \in \mathcal{W}$ which still meets the requirement in (ii), $S$ is actually $c$-dense; i.e., each interval $I \subseteq[0,1]$ contains $c$ points of $S$.

(iii) If $W_{1}, W_{2} \in \mathcal{W}$ with $W_{1} \neq W_{2}$ and $x=x_{W_{1}}, y=x_{W_{2}}$, then there exist arbitrary long finite sequences of consecutive integers $n, n+1, \ldots, N$ and $m, m+$ $1, \ldots, M$ such that $x_{i}=y_{i}=0$ for $i=n, \ldots, N$ and $x_{i}=1$ with $y_{i}=0$ (or $x_{i}=0$ with $\left.y_{i}=1\right)$ for $i=m, \ldots, M$, and such that $x_{m-1}, y_{m-1}$ are either both 0 or both 1.

(2) Let

$$
f(x)= \begin{cases}2 x & \text { if } 0 \leq x \leq \frac{1}{2} \\ 2(1-x) & \text { if } \frac{1}{2} \leq x \leq 1\end{cases}
$$

be the function we discussed in the beginning of this section. Properties (a) and (b) there together with (iii) imply that

$$
\left|f^{n}(x)-f^{n}(y)\right| \leq \frac{1}{2^{N-n}} \quad \text { and } \quad\left|f^{m}(x)-f^{m}(y)\right| \geq 1-\frac{1}{2^{M-m-1}} .
$$

It follows that $S$ is a scrambled set for $f$.

(3) Let $S_{1}$ consist of those $x \in S$ requiring no more than one wedge in their representations; i.e., $x \in S_{1}$ if there exists $r, s \in \Lambda$ such that $x=x_{W_{r s}}$. Let $A_{1}=(\Lambda \times \Lambda) \cap\{(r, s): r<s\}=\{(r, s) \in \Lambda \times \Lambda: r<s\}$. Then $A_{1}$ is a $G_{\delta}$ of cardinality $c$ in the complete space $R_{2}$ and is therefore an absolute $G_{\delta}[\mathbf{O}]$. Then $\left(r_{n}, s_{n}\right) \rightarrow(r, s)$ in $A_{1}$ if and only if $x_{n} \rightarrow x \in S_{1}$, where $x_{n}=x_{W_{r_{n}, s_{n}}}$ and $x=x_{W_{r, s}}$. Thus $S_{1}$ is homeomorphic to $A_{1}$ and is therefore an absolute $G_{\delta}[\mathbf{O}]$. Similarly, if

$$
\begin{aligned}
S_{n}=\{x: & x=x_{W}, \text { where } W=W_{r_{1} s_{1}} \cup \cdots \cup W_{r_{n} s_{n}}, \\
& \text { with } \left.r_{1}<s_{1}<r_{2}<\cdots<r_{n}<s_{n}, \text { consists of } n \text { wedges }\right\},
\end{aligned}
$$

then $S_{n}$ is homeomorphic to $A_{n}=\left\{\left(r_{1}, s_{1}, r_{2}, \ldots, r_{n}, s_{n}\right): r_{1}<s_{1}<\cdots<r_{n}<s_{n}\right.$, and $r_{k}, s_{k} \in \Lambda$ for $\left.k=1,2, \ldots, n\right\}$, and thus $S_{n} \in G_{\delta}$. Since $S=\bigcup_{n=1}^{\infty} S_{n}, S \in G_{\delta \sigma}$. Note each $S_{n}$ is nowhere dense: an $x$ with long strings of 0's and 1's alternating in binary expansion cannot be in $S_{n}$. Hence $S$ is a scrambled, $c$-dense first category $G_{\delta \sigma}$ subset of $[0,1]$.

(4) Let $\left\{I_{n}\right\}$ be an enumeration of the rational intervals in $[0,1]$. For each $n$, the set $S \cap I_{n}$ is $c$-dense in $I_{n}$ and is of type $G_{\delta \sigma}$. Thus, there exists a nonempty perfect set $P_{n} \subseteq S \cap I_{n}$. Let $P=\bigcup_{n=1}^{\infty} P_{n}$. Then $P$ is of type $F_{\sigma}$ and is $c$-dense in $[0,1]$. Furthermore, since $P \subseteq S, P$ is a scrambled set for $f$.

(5) Let $h$ be a homeomorphism of $I_{0}$ onto itself such that $h(P)=T$ has measure one, and $h\left(\frac{1}{2}\right)=\frac{1}{2}$ (such a homeomorphism exists by $[\mathbf{G}]$ ). Let $g=h \circ f \circ h^{-1}$. Then $T$ is a scrambled set for $g$ and $\lambda T=1$. Furthermore $g$ increases on $\left[0, \frac{1}{2}\right]$ and decreases on $\left[\frac{1}{2}, 1\right]$. 
REMARKS. The homeomorphism $h$ can be chosen to be arbitrarily close to the identity. (One only needs to subdivide $I_{0}$ into sufficiently small subintervals and require that $h$ map each subinterval onto itself.) Thus $g$ can be chosen uniformly close to $f$.

Let $S, T$ be the scrambled sets appearing in Theorem 3.1. If $x \in S$, then 0 and $1 / 2^{n}, n=0,1, \ldots$, are the only limit points of $\left\{f^{n}(x)\right\}$. In particular, $S$ contains no points of dense orbit under $f$. Similarly $T$ contains no points of dense orbit of $g$. Let $D_{f}$ and $D_{g}$ denote sets of points having dense orbits under $f$ and $g$ respectively. Then $\lambda S=0, \lambda D_{f}=1, \lambda T=1, \lambda D_{g}=0$.

Furthermore, note that $f$ must be one-to-one on a scrambled set. Similarly, $f^{n}$ must be one-to-one. Thus our example shows that it is possible for a continuous function and all its iterates to be one-to-one on a set of full measure without being one-to-one on $I_{0}$.

4. Category analogues. We say in $\S 3$ that there exists a function $g$ possessing a scrambled set of full measure. The function $g$ we defined is nomadic. Two questions arise naturally:

(1) Does the category analogue of Theorem 3.1 hold? I.e. does there exist a continuous function possessing a scrambled set that is residual in $I_{0}$ ?

(2) What relationships exist between nomadic functions and chaotic functions?

We address both these questions in this section. First we show that if a scrambled set has the Property of Baire, then it must be first category. Since the Property of Baire is the category analogue of a set being measurable, this raises the question of whether a scrambled set can be second category if it lacks the Property of Baire. We show this is possible by showing that every continuous function $f$ with $f^{2}$ nomadic possesses a scrambled set that is second category in every interval. (This statement fails if one weakens the requirement that $f^{2}$ be nomadic to the requirement that $f$ be nomadic.) Finally, we observe in Corollary 4.9 that a continuous $f$ is chaotic if and only if $f^{2}$ is nomadic.

Proposition 4.1. Let $f$ be continuous on an interval $[a, b]$ and let $S$ be a residual subset of $[a, b]$. If $f$ is one-to-one on $S$, then $f(S)$ is residual in $f([a, b])$.

Proof. Let $H$ be a dense set of type $G_{\delta}$ contained in $S$. Then $f(H)$ is a Borel set, being the one-to-one continuous image of a set of type $G_{\delta}$. Thus, if $f(H)$ is not residual in $f([a, b])$, then there exists an open interval $J$ such that $f(H) \cap J$ is first category. Let $I$ be a component interval of the open set $f^{-1}(J)$ and let $H^{*}=H \cap I$. Since $f\left(H^{*}\right)$ is a first category subset of $J$, there exist nowhere dense sets $B_{1}, B_{2}, \ldots$ such that

$$
f\left(H^{*}\right)=\bigcup_{k=1}^{\infty} B_{k} \subseteq \bigcup_{k=1}^{\infty} \bar{B}_{k} \subseteq \bar{J} .
$$

Then $H^{*} \subseteq \bigcup_{k=1}^{\infty}\left(f^{-1}\left(\bar{B}_{k}\right) \cap \bar{I}\right)$. Since $H^{*}$ is residual in $I$, the same is true of $\bigcup_{k=1}^{\infty}\left(f^{-1}\left(\bar{B}_{k}\right) \cap I\right)$. This implies that there is a $k$ such that the closed set $f^{-1}\left(\bar{B}_{k}\right) \cap \bar{I}$ contains an interval $L$. But $H^{*}$ is residual in $L$ and $f$ is one-to-one on $H^{*}$, so the set $f(L)$ is a nondegenerate interval. This is impossible since $f(L) \subset \bar{B}_{k}$, a nowhere dense set. Thus the proof is complete. 
THEOREM 4.2. Let $f: I_{0} \rightarrow I_{0}$ be continuous and let $S$ be a scrambled set for $f$. If $S$ has the Property of Baire, then $S$ is first category.

PROOF. We argue by contradiction. Suppose $S$ is a second category scrambled set possessing the Property of Baire. Then there exists an interval $J$ such that $S$ is residual in $J$. We show first that $f$ must be monotonic on $J$. If not, there exist points $a<b<c$ in $J$ such that $f(a)=f(c) \neq f(b)$. Without loss of generality, assume $\max \{f(x): x \in[a, b]\}=f(b)$. Since $f$ is one-to-one on $S$, it follows from Proposition 4.1 that $f(S \cap[a, b])$ and $f(S \cap[b, c])$ are residual subsets of $f([a, b])$. Thus these two sets have a nonempty intersection, which is impossible since $f$ is one-to-one on $S$. Now let $0<\delta<\frac{1}{2}$. We claim that $f$ is monotonic on $[\delta, 1-\delta]$. Let $x \neq y$ be points of $S \cap J$. Since $S$ is a scrambled set, there exists $n$ such that $\left|f^{n}(x)-f^{n}(y)\right|>1-\delta$, say $f^{n}(x)<\delta$ and $f^{n}(y)>1-\delta$. It now follows from Proposition 4.1 that $f^{n}(S \cap J)$ is residual in $[\delta, 1-\delta]$. If $f$ is not monotonic on $[\delta, 1-\delta]$, then $f$ cannot be one-to-one on the set $f^{n}(S \cap[\delta, 1-\delta])$; i.e., $f^{n+1}$ is not one-to-one on $S \cap[\delta, 1-\delta]$. This is impossible since $S \cap[\delta, 1-\delta]$ is a scrambled set for $f$. Thus the claim is proved. Since $0<\delta<\frac{1}{2}$ is arbitrary, we have shown that $f$ is monotonic on $I_{0}$. However, a monotonic function cannot have scrambled sets. This is a contradiction to the hypothesis that $S$ is a scrambled set for $f$. Thus $S$ must be first category in $I_{0}$.

If we drop the requirement that $S$ have the Property of Baire, the conclusion that $S$ is first category no longer follows. Our next theorem shows this, as well as establishing a connection between nomadic functions and chaotic functions. Observe that the requirement that $f^{2}$ be nomadic implies also that $f$ is nomadic.

THEOREM 4.3. Let $f$ be continuous on $I_{0}=[0,1]$. Suppose $f^{2}$ is nomadic. Assuming the Continuum Hypothesis, there exists a set $S$ of second category in every interval such that if $x, y \in S$ with $x \neq y$, then the set $\left\{f^{n}(x)-f^{n}(y)\right\}$ is dense in $[-1,1]$.

The proof of Theorem 4.3 depends on three propositions that may be of interest in themselves. The authors are grateful to the referee for pointing out that the hypotheses for Proposition 4.4 suffice. Originally we had stronger hypotheses which led to weaker versions of the remaining results.

PROPOSITION 4.4. If $f$ is continuous on $I_{0}=[0,1]$ and $f^{2}$ is nomadic, then to every $\delta>0$ and every open interval $I \subset I_{0}$ there corresponds a positive integer $N$ such that $f^{k}(I) \supset[\delta, 1-\delta]$ for every $k \geq N$.

PROOF. Since $f$ is nomadic, there is a fixed point $x_{0}$ for $f$ in $(0,1)$. Let $J$ be a nondegenerate closed interval such that $x_{0}$ is an end-point of $J$ and $J \subset$ $f\left(\left[0, x_{0}\right]\right) \cap f\left(\left[x_{0}, 1\right]\right) \cap(0,1)$. (If no such interval existed, then $f^{2}\left[0, x_{0}\right]$ would be contained in $\left[0, x_{0}\right]$ and $f^{2}$ would not be nomadic.) For each positive integer $n$, let $J_{n}=f^{n}(J)$. Each set $J_{n}$ is an interval containing $x_{0}$. Now $f$ is nomadic, so there exists a positive integer $M$ such that $f^{M}(J) \supset J$. Let $H=J \cup J_{1} \cup \cdots \cup J_{M-1}$. Then $H$ is an interval containing $J$ and

$$
f(H)=f(J) \cup f\left(J_{1}\right) \cup \cdots \cup f\left(J_{M-1}\right)=J_{1} \cup \cdots \cup J_{M} .
$$

Since $J_{M} \supset J, f(H) \supset H$. 
Now let $H_{k}=f^{k}(H)$. Then the sequence $\left\{H_{k}\right\}$ is an expanding sequence of intervals, $H_{k+1} \supset H_{k}, k=1,2,3, \ldots$. Since each such interval contains $x_{0}$ and since $f$ is nomadic, there exists $M_{1}$ such that $f^{k}(H) \supset[\delta, 1-\delta]$ for all $k \geq M_{1}$.

We now show that there exists $M_{2}$ such that $f^{M_{2}}(I) \supset H$. Since $f$ is nomadic, $O(I)$ has at most two components [BCR, Theorem 10]. But $f^{2}$ is also nomadic, so there must exist a point $z \neq x_{0}, z \in(0,1)$, such that $f(z)=x_{0}$. It follows that some iterate of $f$ maps $I$ onto an interval containing $x_{0}$ and that some later iterate $f^{M_{2}}$ satisfies $f^{M_{2}}(I) \supset H$. Thus $f^{k}(I) \supset[\delta, 1-\delta]$ for all $k \geq N=M_{1}+M_{2}$.

Proposition 4.5. Let $f$ be continuous on $I_{0}$ with $f^{2}$ nomadic. Define a function $g$ on $I_{0} \times I_{0}$ to $I_{0} \times I_{0}$ by $g(x, y)=(f(x), f(y))$. Then there exists a set $A$ that is dense and of type $G_{\delta}$ in $I_{0} \times I_{0}$ such that each point in $A$ has a dense orbit relative to $g$.

ProOF. Let $U=U_{1} \times U_{2}, V=V_{1} \times V_{2}$ be open rectangles whose closures are contained in $(0,1) \times(0,1)$. By Proposition 4.4, there exists a positive integer $N$ such that $f^{N}\left(U_{1}\right) \supset V_{1}$ and $f^{N}\left(U_{2}\right) \supset V_{2}$. Thus $g^{N}(U) \supset V$. Hence the inverse orbit of $V$ under $g$ is dense. Since $V$ is open and $g$ is continuous, this set is also open. Now let $V_{1}, V_{2}, \ldots$ be a countable base for the topology of $(0,1) \times(0,1)$ with $\bar{V}_{i} \subseteq(0,1) \times(0,1)$. Let $A=\bigcap_{k=1}^{\infty} O^{-1}\left(V_{k}\right)$, where $O^{-1}\left(V_{k}\right)$ is the inverse orbit of $V_{k}$ under $g$. Then $A$ is a dense $G_{\delta}$ and each point of $A$ has a dense orbit relative to $g$.

Proposition 4.6. Let $f$ be continuous on $I_{0}$ with $f^{2}$ nomadic. Then there exists a residual set $B$ such that if $x \in B$, there exists a residual set $B_{x} \subset B$ such that if $y \in B_{x}$ with $y \neq x$, then the set $\left\{f^{n}(y)-f^{n}(x)\right\}$ is dense in $[-1,1]$.

Proof. Let $A$ be the set of Proposition 4.5. By the Kuratowski-Ulam Theorem [O], there exists a residual set $B \subset I_{0}$ such that if $x \in B$, then $B_{x}=\{y:(x, y) \in A\}$ is a dense $G_{\delta}$ in $I_{0}$. But, if $(x, y) \in A$, then the set $g^{n}(x, y)$ is dense in $I_{0} \times I_{0}$ by Proposition 4.5. This implies that $\left\{f^{n}(x)-f^{n}(y)\right\}$ is dense in $(-1,1)$. Since $B$ and $B_{x}$ are residual sets, so is $B_{x} \cap B$, so there is no loss of generality in assuming $B_{x} \subset B$.

We are now ready for the proof of Theorem 4.3.

PROOF OF THEOREM 4.3. Let $B$ and $B_{x}$ have the meanings indicated in Proposition 4.6. We assume the Continuum Hypothesis. Let $\Omega$ be the first uncountable ordinal. Well-order the second category sets of type $G_{\delta}$ in $I_{0}$ by $\Omega$ :

$$
G_{0}, G_{1}, \ldots, G_{\alpha}, \ldots, \quad \alpha<\Omega .
$$

Let $x_{0} \in G_{0} \cap B$, and choose $x_{1} \neq x_{0}, x_{1} \in G_{1} \cap B_{x_{0}}$. Suppose we have chosen $x_{\beta}$ for all $\beta<\alpha$ such that $x_{\beta} \neq x_{\gamma}$ for $\gamma<\beta, x_{\beta} \in G_{\beta} \cap B_{x_{\gamma}}$ for all $\gamma<\beta$. Consider the set $S_{\alpha}=\left(G_{\alpha} \cap \bigcap_{\gamma<\alpha} B_{x_{\gamma}}\right)-\left\{x_{\beta}: \beta<\alpha\right\}$. This set is a countable intersection of dense sets of type $G_{\delta}$, thus $S_{\alpha}$ is also a dense $G_{\delta}$. Let $x_{\alpha} \in S_{\alpha}$ and let $S=\left\{x_{\alpha}: \alpha<\Omega\right\}$. We show that $S$ has the desired properties.

To see $S$ is second category in every interval, we need only note that $S$ intersects every second category set of type $G_{\delta}$. (If $S$ were first category in some interval $I$, then $I-S$ would be residual in $I$ and would therefore contain a $G_{\delta}$ set dense in $I$. This set would be a second category $G_{\delta}$ missing $S$.) Now, let $x, y \in S$, say $x=x_{\alpha}$, $y=x_{\beta}$ with $\alpha<\beta$. Then $y \in B_{x}$ so $\left\{f^{n}(y)-f^{n}(x)\right\}$ is dense in $[-1,1]$ as required. 
REMARK. Let $D=\{x: O(x)$ is dense $\}$. Then $D$ is a dense $G_{\delta}$. Let $T=D \cap S$. Then $T$ is second category in every interval and is scrambled for $f$. If $p$ is a periodic point for $f$, then $\lim \sup _{n \rightarrow \infty}\left|f^{n}(p)-f^{n}(x)\right|>0$ for every $x \in T$. This condition is sometimes part of the requirement of a scrambled set $[\mathbf{K}, \mathbf{S 1}]$.

REMARK. Propositions 4.5 and 4.6 and Theorem 4.3 are not valid in general without the assumption that $f^{2}$ be nomadic. One need only consider a nomadic function with a cut-point $[\mathbf{B C R}]$, that is a point $z$ such that $f([0, z])=[z, 1]$ and $f([z, 1])=[0, z]$ and $O^{-1}(z)=z$. It is clear that if $x<y<z$ for such a function, then $\lim _{n \rightarrow \infty} \sup \left|f^{n}(y)-f^{n}(x)\right|<1$.

Nonetheless, in that case, weaker conclusions are possible since the function $f^{2}$ is nomadic on $[0, z]$ and on $[z, 1]$ and has infinitely many fixed points. (See Theorem 11 of [BCR] for some results concerning behavior of nomadic functions with cut points.) Thus $f^{2}$ exhibits chaotic-like behavior on each of the two intervals. More specifically, $f^{2}$ exhibits the behavior of Theorem 4.3 on each of these intervals. In particular, if $x$ and $y$ are in $[0, z]$, then $\left\{f^{n}(x)-f^{n}(y)\right\}$ are dense in $[-z, z]$ and have similar behavior for $x, y \in[z, 1]$.

Theorem 4.3 showed that continuous functions with $f^{2}$ nomadic are chaotic. We now obtain a converse.

THEOREM 4.7. Let $f: I_{0} \rightarrow I_{0}$ be continuous. If there exists a set $S$, dense in $I_{0}$ such that $\lim \sup _{n \rightarrow \infty}\left|f^{n}(x)-f^{n}(y)\right|=1$ for all $x \neq y$ in $S$, then $f^{2}$ is nomadic.

PROOF. Let $K$ be a compact set with interior points such that $f^{2}(K) \subset K$. We show $K=I_{0}$. This implies that $f^{2}$ is nomadic [BCR]. Let $U$ be an interval contained in $K$, let $x, y \in S \cap U$ and let $\varepsilon>0$. Choose $\delta$ such that $0<\delta<\varepsilon$ and $f([\delta, 1-\delta]) \supset[\varepsilon, 1-\varepsilon]$. By the definition of $S$, there is a positive integer $N$ such that $\left|f^{N}(x)-f^{N}(y)\right|>1-\delta$. Thus $f^{N}(U) \supset[\delta, 1-\delta] \supset[\varepsilon, 1-\varepsilon]$ and $f^{N+1}(U) \supset f([\delta, 1-\delta]) \supset[\varepsilon, 1-\varepsilon]$ because of the definition of $\delta$. One of the numbers $N$ or $N+1$ is even, say $N$. Then since $U \subset K, K \supset f^{N}(U) \supset[\varepsilon, 1-\varepsilon]$.

This inclusion $K \supset[\varepsilon, 1-\varepsilon]$ is valid for every $\varepsilon>0$, thus $K=I_{0}$. Thus the only compact invariant set for $f^{2}$ with interior is $I_{0}$, as was to be shown.

Combining Theorems 4.3 and 4.7 we obtain Theorem 4.8 .

THEOREM 4.8. Assume the Continuum Hypothesis. Let $f$ be continuous on $I_{0}$. Then there exists a dense set $S$ such that for $x, y \in S(x \neq y)$,

$$
\limsup _{n \rightarrow \infty}\left|f^{n}(x)-f^{n}(y)\right|=1
$$

if and only if $f^{2}$ is nomadic. In that case there is a set $T$ of second category in every interval such that for $x, y \in T(x \neq y)$, the set $\left\{f^{n}(x)-f^{n}(y)\right\}$ is dense in $[-1,1]$.

In particular, we obtain the following corollary that relates nomadicity to chaos.

COROLlARY 4.9. Under the Continuum Hypothesis, a continuous function $f$ defined on $I_{0}$ is chaotic if and only if $f^{2}$ is nomadic.

\section{REFERENCES}

[BCR] A. M. Bruckner, J. Ceder, and M. Rosenfeld, On invariant sets for functions, Bull. Inst. Math. Acad. Sinica 3 (1975), 333-347.

[G] W. Gorman, The homeomorphic transformation of c-sets into d-sets, Proc. Amer. Math. Soc. 17 (1966), 825-830. 
[K] I. Kan, A chaotic function possessing a scrambled set with positive Lebesgue measure, Proc. Amer. Math. Soc. 92 (1984), 45-49.

[LY] Tien-Yien Li and James A. Yorke, Period three implies chaos, Amer. Math. Monthly 82 (1975), 985-992.

[O] John Oxtoby, Measure and category, Springer-Verlag, New York, 1980.

[S1] J. Smital, A chaotic function with some extremal properties, Proc. Amer. Math. Soc. 87 (1983), 54-56.

[S2] _ A chaotic function with a scrambled set of positive measure, Proc. Amer. Math. Soc. 92 (1984), 50-54.

[VSK] E. B. Vul, Ya. G. Sinai, and K. M. Khanin, Feigenbaum Universality and the thermodynamic formalism, Russian Math. Surveys 39 (1984), 1-40.

Department of MAthematics, University of CAlifornia, Santa Barbara, CALIFORNIA 93106

Department of Mathematics, TAMkang University, TAMsui, TAipei, TAiWAN, REPUBLIC OF CHINA 